\title{
O Conhecimento Tecnológico Pedagógico do Conteúdo no Contexto Lusófono: uma revisão sistemática da li- teratura
}

The Technological Pedagogical Content Knowledge in Lusophone Context: a systematic review of the literature

\author{
Luiz Gustavo Ribeiro Rolando \\ LAEFiB - Instituto Oswaldo Cruz. \\ Av. Brasil, 4365 Manguinhos \\ 21040-360 Rio de Janeiro - RJ - \\ Brasil \\ gustavor@ioc.fiocruz.br
}

\author{
Maurício Roberto Motta Pinto da Luz \\ LAEFiB - Instituto Oswaldo Cruz. \\ Av. Brasil, 4365 Manguinhos 21040-360 \\ Rio de Janeiro - RJ - Brasil \\ mauluz@ioc.fiocruz.br
}

\author{
Daniel Fábio Salvador \\ Fundação Cecierj. \\ Rua da Ajuda, n 5 Centro \\ 20040-000 Rio de Janeiro - RJ \\ - Brasil \\ salvador@cecierj.edu.br
}

\begin{abstract}
Resumo O ensino é caracterizado pelo alto nível de variabilidade de situações enfrentadas pelo professor e por uma interconexão entre teoria e prática. Dessa forma, requer dos professores o entrelaçamento de diferentes tipos de conhecimento aplicáveis em diferentes situações. O objetivo do presente estudo é identificar e sintetizar resultados de pesquisa no contexto Lusófono acerca da integração de tecnologia no ensino à luz do modelo teórico Conhecimento Tecnológico Pedagógico do Conteúdo (CTPC). Os resultados obtidos e discutidos indicam que a pesquisa nessa área encontra-se em um estágio inicial no contexto dos países de língua portuguesa. A pesquisa empírica sobre o modelo CTPC se beneficiaria da utilização de instrumentos validados, o que permitiria o cotejamento de resultados obtidos com aqueles de outros países. Esse tipo de paralelo contribuiria para uma melhor compreensão e reorientação das políticas públicas voltadas para a utilização de tecnologias educacionais no ensino.
\end{abstract}

Palavras-Chave: Conhecimento Tecnológico Pedagógico do Conteúdo, CTPC, Ensino, Tecnologias de Informação e Comunicação, TICs, Tecnologia

\begin{abstract}
Teaching is characterized by a high level of variability of situations faced by teachers and an interconnection between theory and practice. Thus, requires teachers interleaving different types of knowledge to be applied in different situations. The aim of this study is to identify and synthesize results of research in Lusophone context about technology integration in teaching based on the theoretical model Technological Pedagogical Content Knowledge (TPACK). The results was discussed and indicate that research area is in early stage. Empirical research on the TPACK model would benefit from the use of validated instruments, which would allow the comparison of results obtained in the context Lusophone with those of other countries. This type of parallel contribute to a better understanding and eventual reorientation of the various public policies for the use of educational technologies in teaching.
\end{abstract}

Keywords: Technological Pedagogical Content Knowledge, TPACK, Teaching, Information and Communications Technology, ICT, Technology 


\section{Introdução}

A atual sociedade é influenciada pela informatização e pelo paradigma tecnológico, caracterizado pela alta velocidade em que a informação é gerada, processada e compartilhada [1], [2]. As tecnologias de informação e comunicação (TICs), enquanto ferramentas que permitem o acesso, a transformação e a produção de informação, que pode estar em formato de texto, imagem, som, dados, documentos multimídia e hipermídia, se constituem numa linguagem de comunicação essencial no século XXI [3][5]. Recentemente, com a popularização da Internet e o avanço tecnológico, surgem a todo o momento mais ferramentas digitais e a tendência de utilizá-las para fins educacionais [6], possibilitando novos processos de ensino e aprendizagem [5], [7], [8].

Instituições internacionais como a British Educational Communications and Technology Agency [9], [10] e a Organização das Nações Unidas para a Educação a Ciência e a Cultura [11], [12] recomendam o uso das TICs no ensino e aprendizagem. Seguindo estas orientações, estudos indicam que elas podem ser utilizadas para apoiar e favorecer a aprendizagem, criar situações baseadas em problemas do mundo real na sala de aula, como oportunidades de feedback e reflexão, na construção de comunidades de aprendizagem, além de expandir as possibilidades de formação continuada de professores [13]-[16].

Balanskat, Blamire, e Kefala [17] apontam que o uso de TICs no ensino tem forte efeito motivacional, especialmente no ensino básico, pois os alunos prestam mais atenção durante as aulas assumindo maior responsabilidade pela sua própria aprendizagem. Segundo Osborne e Hennessy [18], o uso de TICs nas aulas de ciências traz diversos benefícios para os estudantes, tais como desenvolvimento da habilidade de pensamento crítico, de manipulação e coleta de dados, bem como o aumento do acesso ao conhecimento apresentado em formato visual, da motivação e do engajamento. Corroborando esses achados, Lee et al. [14] realizaram revisão de literatura sobre a aprendizagem de ciências baseada na Internet. Segundo esses autores, o uso de ambientes virtuais de aprendizagem gera melhorias no domínio afetivo dos estudantes, atitudes e motivação; bem como nas habilidades de argumentação científica, de interpretação e de processamento de dados. Também no Brasil, documentos oficiais refletem a importância da utilização tecnologias na educação [19], [20]. Nesse sentido, a Conferência Nacional de Educação reiterou a importância do uso de tecnologias no ensino, bem como a efetivação de uma política de formação de professores para o seu uso [20], [21]. No entanto, estudos empíricos recentes indicam que professores no Brasil fazem pouco uso de tecnologias na sua prática docente [22]-[24].

Segundo Mishra e Koehler [25], ensinar não é uma tarefa simples, pois consiste em habilidade cognitiva complexa, que ocorre em um ambiente dinâmico e pouco estruturado. $\mathrm{O}$ ensino é caracterizado pelo alto nível de variabilidade de situações enfrentadas pelo professor e por uma interconexão entre teoria e prática, que é dependente do contexto. Dessa forma, o ato de ensinar com o uso de tecnologia requer dos professores o entrelaçamento de diferentes tipos de conhecimento para se aplicar em diferentes situações, que por sua vez, envolvem diversas estruturas conceituais, perspectivas novas e originais, mesmo em casos que podem parecer superficialmente semelhantes [25]-[27]. Os professores lidam com situações complexas na sala de aula e, para lidar com elas, precisam de um conjunto de conhecimentos que sustente os processos de tomada de decisão para uma atuação efetiva em situações específicas de ensino e aprendizagem com o uso de tecnologias [25]-[27].

\subsection{O modelo teórico Conhecimento Tecnoló- gico, Pedagógico do Conteúdo}

Ampliando o modelo proposto por Shulman [28], no qual a formação do professor deveria integrar o conhecimento pedagógico com o aprofundamento no conhecimento do conteúdo, Mishra e Koehler [25] propuseram a inclusão de um terceiro tipo de conhecimento a ser considerado ao se referir às bases de conhecimento do professor, o conhecimento tecnológico. Fundamentado na ideia de Shulman, de que tópicos específicos de ensino poderiam ser representados por meio de analogias, ilustrações, exemplos, explicações e demonstrações de modo a tornar um conteúdo compreensível para outros, Mishra e Koehler alegaram que as tecnologias poderiam desempenhar um papel crítico em cada um desses aspectos, tendo o potencial de mudar a natureza da sala de aula, na medida em que proporcionam uma série de possibilidades de representações e demonstrações que podem ajudar a tornar o conteúdo mais acessível para o aluno [25].

A adição da base de conhecimento tecnológico ao conceito de conhecimento pedagógico do conteúdo proporcionou uma abordagem completamente nova da forma de tratar a questão da integração da tecnologia no ensino, uma vez que, o "bom ensino" requereria o entendimento de como a tecnologia se integraria com o conteúdo e a pedagogia [25]-[27]. Assim, o modelo acerca das bases de conhecimento do professor passou a se apoiar no tripé composto dos eixos pedagógico, tecnológico e conteúdo (Conhecimento Tecnológico, Pedagógico do Conteúdo CTPC, do inglês Technological, Pedagogical, Content, 
Knowledge - TPACK). Esse modelo propõe o entrelaçamento dessas três bases de conhecimentos (Figura 1).

Cada um dos três círculos representa uma das três bases de conhecimento (conteúdo $[\mathrm{C}]$, pedagogia $[\mathrm{P}]$ e tecnologia $[\mathrm{T}]$ ), cujas superposições parciais, resultam em três novas bases de conhecimento, além do conhecimento pedagógico do conteúdo, proposto por Shulman. As três novas bases estão relacionadas ao papel desempenhado pela adição da tecnologia no ensino. A seguir, as sete bases de conhecimento são definidas de acordo com Mishra e Koehler [25].

Conhecimento do conteúdo (CC): é o conhecimento sobre o conteúdo que deve ser aprendido ou ensinado. Os conteúdos a serem cobertos nas escolas diferem quanto às diferentes disciplinas (matemática, história, ciências entre outras), quanto a um tópico dentro de uma mesma disciplina (transformação de energia ou genética, em Biologia), bem como em relação ao nível de ensino (fundamental, médio ou superior). Professores devem conhecer e entender o conteúdo que lecionam, incluindo o conhecimento dos fatos centrais, conceitos, teorias, e procedimentos de um determinado campo do saber.

Conhecimento pedagógico (CP): é o conhecimento sobre os processos, práticas e métodos de ensino e aprendizagem, e como eles envolvem os objetivos, valores e propósitos educacionais em geral. É uma forma genérica de conhecimento que está envolvida em todas as questões de aprendizagem dos alunos, gestão de sala de aula, desenvolvimento de plano de aula, implementação e avaliação dos alunos. Ele inclui o conhecimento sobre as técnicas ou métodos para se utilizar na sala de aula; a natureza do público-alvo e estratégias para avaliar a compreensão dos alunos. Um professor com profundo conhecimento pedagógico entende como os alunos constroem conhecimento, adquirem habilidades, desenvolvem hábitos mentais e disposições positivas em relação à aprendizagem.

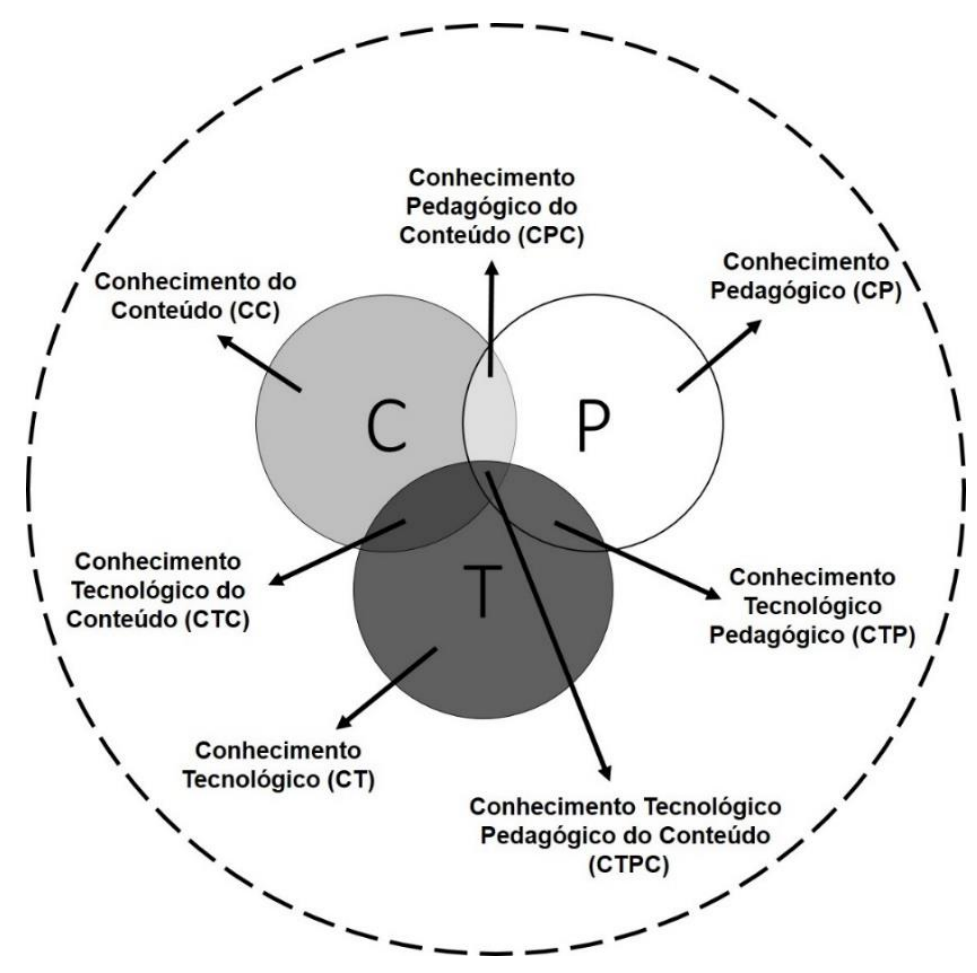

Figure 1: Modelo teórico CTPC

Conhecimento pedagógico do conteúdo (CPC): é o conhecimento que mistura o conhecimento do conteúdo e o conhecimento pedagógico em um entendimento de como tópicos específicos, problemas ou questões são organizados, representados, e adaptados para os diversos interesses e habilidades dos alunos, e apresentados para instrução [28]. Este conhecimento inclui saber quais abordagens de ensino melhor se ajustam a um conteúdo específico, e da mesma forma, saber como elementos deste conteúdo podem ser organizados para um ensino melhor. Este conhecimento é diferente do conhecimento de um expert daquela área do saber, mas também é diferente do conhecimento pedagógico geral compartilhado pelos professores em todas as disciplinas. O CPC está relacionado com a representação e formulação de conceitos, técnicas pedagógicas, conhecimento do que faz determinados conceitos difíceis ou 
fáceis de se aprender, o conhecimento acerca do conhecimento prévio que os alunos possuem, e teorias epistemológicas. Também envolve o conhecimento de estratégias de ensino que incorporem apropriadas representações conceituais, a fim de enfrentar as dificuldades e erros conceituais dos alunos.

Conhecimento Tecnológico (CT): é o conhecimento sobre as tecnologias habituais da sala de aula, tais como livros, giz e quadro-negro, e tecnologias mais avançadas, tais como a Internet e vídeo. Isso envolve as habilidades necessárias para operar tecnologias específicas. No caso das tecnologias digitais, inclui o conhecimento de sistemas operacionais e computador, bem como a capacidade de usar software, tais como processadores de texto (Word), planilhas eletrônicas (Excel), navegadores (Google Chrome) e e-mail. O CT inclui o conhecimento de como instalar e remover dispositivos periféricos, instalar e remover programas de software, além de criar e arquivar documentos. Como a tecnologia está continuamente mudando, a natureza do CT também precisa mudar com o tempo. A capacidade de aprender e adaptar-se às novas tecnologias é importante.

Conhecimento Tecnológico do Conteúdo (CTC): é o conhecimento sobre a forma como tecnologia e conteúdo estão reciprocamente relacionados. Novas tecnologias podem proporcionar novas e variadas formas de representação e maior flexibilidade para navegar através destas representações. Os professores precisam saber não apenas o conteúdo que ensinam mas também a maneira pela qual esse conteúdo pode ser modificado através da aplicação da tecnologia. Por exemplo, considere o programa Geometer's Sketchpad como ferramenta de ensino da geometria. Ele permite que os alunos joguem com as formas e modelos, tornando mais fácil construir padrões geométricos. Este tipo de aprendizagem da geometria não estava disponível antes dessa tecnologia. Argumentos semelhantes podem ser feitos para inúmeros outros softwares.

Conhecimento Tecnológico Pedagógico (CTP): é o conhecimento sobre como as diferentes tecnologias, seus componentes e suas capacidades, são utilizadas no contexto de ensino e aprendizagem, e por outro lado, saber como o ensino pode mudar, como resultado do uso dessas determinadas tecnologias. Isso pode incluir o entendimento de que existe uma série de ferramentas para uma determinada tarefa, bem como a habilidade de escolher uma única ferramenta com base na sua função. Ou ainda escolher uma ferramenta por permitir seu uso de variadas formas ou com variadas estratégias. Inclui também o conhecimento de estratégias pedagógicas e a capacidade de aplicar essas estratégias para o uso de tecnologias. Isso inclui o conhecimento de ferramentas para manutenção de registros de classe, frequência e atribuição de notas, e o conhecimento de outras ferramentas mais genéricas, tais como WebQuests, fóruns de discussão e salas de bate- papo.

Conhecimento Tecnológico Pedagógico do Conteúdo (CTPC): é uma forma de conhecimento emergente que vai além de todas as três bases (de conteúdo, pedagógica, e tecnológica). Este conhecimento é diferente do conhecimento de um expert em tecnologia ou de outro especialista em uma determinada área do saber humano e difere também do conhecimento pedagógico geral compartilhado por professores de diferentes disciplinas. CTCP é a base do "bom ensino" com tecnologia e requer uma compreensão da representação de conceitos utilizando tecnologias; técnicas pedagógicas que as utilizem de maneira construtiva para ensinar o conteúdo; conhecimento do que torna conceitos difíceis ou fáceis de serem aprendidos e de como a tecnologia pode ajudar a resolver alguns dos problemas que os alunos enfrentam; o conhecimento acerca do conhecimento prévio que os alunos possuem, e teorias epistemológicas; conhecimento de como tecnologias podem ser usadas para construir o conhecimento existente e desenvolver novas epistemologias ou fortalecer as antigas.

O modelo CTPC se tornou, na última década, um dos mais importantes referenciais teóricos para a pesquisa acerca da integração de tecnologia no ensino em todo o mundo [27], [29], [30]. Entretanto pouco se sabe sobre sua perfusão em países de língua portuguesa, uma vez que, estudos de revisão sistemática, recentemente publicados em língua inglesa, não apresentaram estudos relacionados ao contexto Lusófono [29], [30], [31], [32]. Nossa hipótese é a de que, embora a integração das TICs seja considerada importante fator para a educação no século XXI e que documentos oficiais recomendem seu uso no ensino, o campo de pesquisa que trata desta temática no contexto Lusófono está pouco inserido no diálogo que vem se desenvolvendo no mundo. Desta forma, o entendimento do estado em que se encontra a aplicação do modelo CTPC no contexto lusófono poderá ser útil a pesquisadores, no sentido de apontar lacunas e indicar focos para a pesquisa futura.

\section{Metodologia}

A metodologia empregada neste estudo está baseada nos procedimentos de síntese de pesquisa propostos por Sampaio e Mancini [33], Cooper e Hedges [34] e Kitchenham [35]. Uma revisão sistemática de literatura serve para indicar novos rumos para futuras investigações e identificar métodos de pesquisa utilizados em uma área de estudo [33-35]. Os procedimentos metodológicos neste tipo de pesquisa incluem a formulação de perguntas de investigação, a definição da estratégia de busca, o estabelecimento de critérios de inclusão e exclusão de documentos, e a análise criteriosa da qualidade da literatura selecionada [33-35]. 


\subsection{Perguntas de investigação}

O objetivo do presente estudo é identificar e sintetizar resultados de pesquisa no contexto Lusófono acerca da integração de tecnologia no ensino à luz do modelo teórico Conhecimento Tecnológico Pedagógico do Conteúdo (CTPC). Para isso, pretendemos responder as seguintes perguntas de investigação: i) em que extensão o modelo CTPC está incorporado à produção científica acerca da integração da tecnologia no ensino no contexto Lusófono? ii) quais os instrumentos e ou métodos de avaliação acerca das bases de conhecimento envolvidas no modelo CTPC estão disponíveis em língua portuguesa?

\subsection{Processo de busca}

A busca realizada na literatura foi conduzida em nove diferentes bases de dados, a saber: ACM Digital Library, Scopus, Science Direct, Web of Science, IEEExplore, SpringerLink, Education Resources Information Center (ERIC), SciELO e Google Acadêmico. Utilizou-se o ano de 2014 como limite superior para a busca nas bases de dados em relação ao período de publicação. Assim, qualquer documento publicado até o ano de 2014 foi, ao menos inicialmente, considerado.

Os termos de busca utilizados foram "Technological Pedagogical Content Knowledge", "TPACK", "TPCK", e suas combinações "TPACK" and "Technological Pedagogical Content Knowledge", "TPCK" and "Technological Pedagogical Content Knowledge". Para complementar a busca foi utilizado o termo "conhecimento tecnológico pedagógico do conteúdo", tradução para a língua portuguesa do termo original em inglês.

\subsection{Critérios de inclusão e exclusão}

A busca na literatura gerou um resultado inicial de 674 documentos, dos quais 187 eram repetições. Os itens repetidos foram eliminados, restando assim 487 documentos, aos quais foram aplicados os critérios de inclusão e exclusão para compor a amostra final de documentos analisados.

Foram utilizados dois critérios objetivos para selecionar a amostra final de documentos: a) estar redigido em língua portuguesa e b) tratar-se de artigo publicado em periódico científico ou anais de congresso científico. A utilização desses critérios de inclusão resultaram em um conjunto de 60 documentos que compõem a amostra analisada no presente estudo. Cabe ressaltar que a eliminação da maior parte dos documentos incialmente encontrados se deveu ao foco deste estudo ser direcionado para a produção científica em língua portuguesa, especificamente do contexto Lusófono. Um dos estudos escrito em língua portuguesa, cujo contexto de aplicação é os Estados Unidos, foi eliminado da análise. Entre os 487 documentos iniciais foram encontradas quatro revisões sistemáticas de literatura, redigidas em língua inglesa nas quais não se encontram qualquer um dos artigos analisados nesta pesquisa. Essas revisões de literatura foram utilizadas para discutir os resultados obtidos no presente estudo.

\subsection{Análise dos dados}

Os 60 artigos foram analisados segundo as seguintes características da publicação:

- Ano de publicação;

- Local de publicação (periódico científico ou anais de congresso);

- Local do estudo (Brasil ou Portugal);

- Tipo de estudo (empírico ou teórico).

Todos os estudos selecionados, que se basearam em observação e ou experimentação, que por sua vez, geraram dados utilizados como resultados, foram classificados como do tipo empírico. Os artigos classificados como do tipo empírico, foram analisados também segundo duas categorias, Delineamento Metodológico e Nível de Utilização do Modelo CTPC.

A categoria de Delineamento Metodológico foi utilizada de forma a explorar as características gerais dos estudos. Essas características são baseadas em Chai, Koh e Tsai [31] e Koelher, Shin e Mishra [32] a saber:

- Abordagem metodológica;

- Metodologia de pesquisa;

- Área de conhecimento;

- Público alvo/nível de ensino;

- Tipo de intervenção.

Já a categoria Nível de Utilização do Modelo CTPC, foi elaborada pelos autores deste estudo, de forma a identificar a aplicação do modelo teórico CTPC nos artigos analisados, como se segue:

- Uso teórico (cita o modelo CTPC exclusivamente na seção de introdução e ou discussão como referencial teórico para a integração de tecnologia no ensino).

- Uso metodológico (utiliza ou propõe instrumento de avaliação e ou metodologia de análise para avaliar as bases de conhecimento de professores à luz do modelo CTPC).

As categorias utilizadas na presente pesquisa foram definidas de forma consensual pelos autores do estudo. Após discussão sobre o significado de cada item, uma amostra que compreende cerca de $20 \%$ dos artigos foi categorizada independentemente por dois codificadores. Foi obtido um acordo de $99 \%$ entre os dois codificadores. Devido ao ele- 
vado índice de acordo alcançado, os artigos restantes foram categorizados por um codificador. Nos poucos casos em que ocorreram dúvidas, a categorização foi realizada utilizando os dois codificadores de forma a alcançar consenso. Os dados foram coletados e armazenados em uma planilha de Excel. O apêndice 1 apresenta a lista dos 60 artigos, cada um com o respectivo código identificador.

\section{Resultados}

Os procedimentos sistemáticos de revisão de literatura utilizados neste estudo resultaram em uma amostra de 60 artigos, publicados em língua portuguesa e relacionados ao contexto Lusófono. Ao analisarmos a relação do número de artigos publicado por ano de publicação, observa-se uma forte tendência de crescimento da produção científica que tem alguma relação com o modelo teórico CTPC (Figura 2). Tomando como ponto de partida o ano de 2006, data de publicação do artigo seminal de Mishra e Koelher, observa-se que pesquisadores começam, progressivamente, a referenciar o modelo CTPC em seus estudos. Dos 60 artigos selecionados para esta revisão de literatura, $45 \%$ foram publicados em periódicos científicos e $55 \%$ em anais de congresso. Em relação ao local do estudo, $65 \%$ dos artigos dizem respeito ao contexto brasileiro e $35 \%$ ao contexto português. Já em relação ao tipo de estudo, $70 \%$ são estudos empíricos e $30 \%$ teóricos.

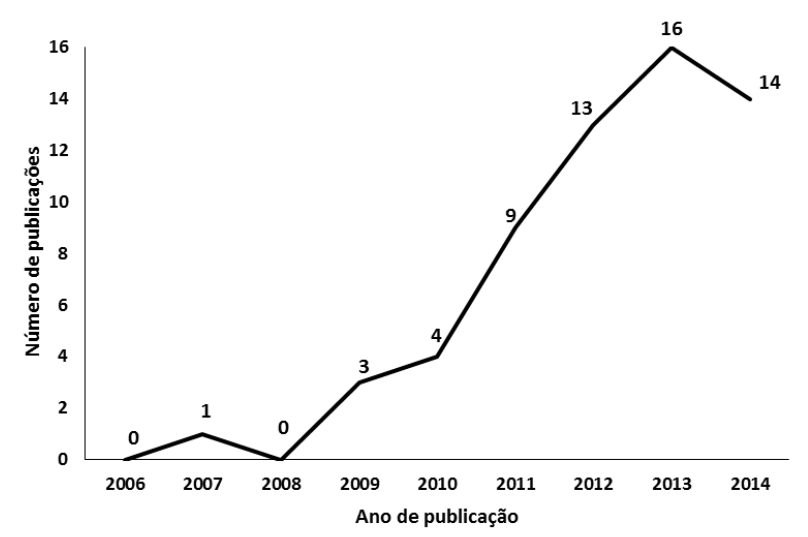

Figura 2: Crescimento do número de publicações ( $\mathrm{n}=60$ artigos).

\subsection{Artigos teóricos}

Os 18 artigos classificados como teóricos são listados no apêndice 1. De forma geral, esses artigos relacionam o modelo CTPC com a questão da integração da tecnologia no ensino. Treze deles $(72,2 \%)$ fazem menção ao modelo CTPC em parte do texto, como um referencial teórico para o ensino em geral (A05), para o ensino de alguma matéria específica por meio de tecnologia, como por exemplo a matemática (A29) ou para a formação de professores (A02, A12), indicando que esses profissionais poderiam se beneficiar com a formação para a inclusão de tecnologias no seu ensino.

Cinco artigos (A09, A26, A41, A42 e A47), se dedicam a apresentar detalhadamente o modelo CTPC, como referencial teórico capaz de revolucionar o processo de desenvolvimento profissional de professores. Coutinho (A09) discute a formação de professores em Portugal para o uso de TICs e conclui afirmando que programas de formação devem passar necessariamente pelo desenho de modelos que vão ao encontro do desenvolvimento integrado das competências docentes de acordo com o referencial do CTPC. Na mesma linha de argumentação, Sampaio e Coutinho (A42), abordam problemas relacionados a integração de tecnologias no ensino e indicam que programas de formação de professores para o uso de tecnologias ignoram a variação inerente das diferentes formas de conhecimento disciplinar. As autoras alegam que o desenvolvimento das bases de conhecimento envolvidas no modelo CTPC deveria ser organizado em torno de conteúdos específicos.

Em consonância com Sampaio e Coutinho (A47), Palis (A26) discute a contextualização do conhecimento pedagógico do conteúdo no ensino de matemática. $\mathrm{O}$ autor se reporta a estudos empíricos internacionais que relacionaram o modelo CTPC ao ensino de matemática e exemplifica teoricamente como o modelo CTPC pode ser desenvolvido no currículo, avaliação, aprendizagem e acesso. Por fim, entre os artigos teóricos, se destaca o artigo de Sampaio e Coutinho (A41) que discute, à luz de estudos empíricos, a avaliação das bases de conhecimento envolvidas no modelo CTPC. Segundo as autoras é necessário estabelecer a triangulação metodológica entre diferentes instrumentos de avaliação baseados não somente em auto relato dos professores, mas também na planificação das suas aulas e na análise propriamente dita das mesmas. Nesse sentido, as autoras traduzem para a língua portuguesa uma rubrica de avaliação de integração tecnológica em planos de ensino baseada no modelo CTPC. Essa rubrica foi criada no contexto americano e foi validada somente na língua inglesa.

\subsection{Artigos empíricos}

Os 42 artigos classificados como empíricos também são listados no apêndice 1. A análise desses artigos levou em consideração a forma como o modelo CTPC foi abordado e o delineamento metodológico a partir do qual as pesquisas foram realizadas. Os itens analisados em relação ao delineamento metodológico são apresentados na tabela 1. Apenas dois artigos utilizaram abordagem quantitativa (A38 e A48), nos quais os autores utilizaram testes estatísticos de inferência. Salvador, Rolando e Rolando (A38) compararam estatisticamente os resultados de evasão em diferentes cursos online, enquanto Silva et al (A48) compararam professores universitários do Brasil e de Portugal, quanto ao seu letramento digital. Os demais 40 artigos utilizaram análises qualitativas dos dados coletados por meio 
de variados métodos. Destaca-se a utilização de questionários e entrevistas nos quais se buscou entender como as tecnologias foram utilizadas em contextos escolares específicos (A20, A25, A33 e A45) ou como professores percebem a tecnologia na sua prática docente (A16 e A24). Outros métodos utilizados foram estudos de caso, descritivo-analíticos, pesquisa-ação, grupo focal e observação.

No que tange à especificidade das áreas de conhecimento, 40,5\% dos estudos estão ligados aos conteúdos de Matemática, indicando que o modelo CTPC possui maior perfusão nesse campo do ensino. Esses estudos, em sua maioria, estavam relacionados ao uso de computadores/tablets (A03, A04 e A11) e softwares, principalmente, os de geometria (A03, A08, A21 e A35). No campo das ciências naturais e saúde destaca-se a relação entre o modelo CTPC e a integração da tecnologia no ensino por meio de ambientes virtuais de aprendizagem, tanto em disciplinas de ensino superior no campo da saúde (A15, A16 e A17), quanto no campo da formação continuada de professores de Biologia (A38 e A39).

\begin{tabular}{|c|c|c|c|}
\hline \multicolumn{2}{|c|}{ Delineamento metodológico } & \multirow{2}{*}{$\begin{array}{l}\mathrm{N}^{\circ} \\
40 \\
\end{array}$} & \multirow{2}{*}{$\frac{\%}{95,2 \%}$} \\
\hline \multirow{2}{*}{ Abordagem } & Qualitativa & & \\
\hline & Quantitativa & 2 & $4,8 \%$ \\
\hline \multirow{4}{*}{$\begin{array}{l}\text { Metodologia } \\
\text { de pesquisa }\end{array}$} & $\begin{array}{l}\text { Entrevista e/ou } \\
\text { questionário }\end{array}$ & 17 & $40,5 \%$ \\
\hline & Estudo de caso & 6 & $14,3 \%$ \\
\hline & Descritivo-analítico & 5 & $11,9 \%$ \\
\hline & Outros & 14 & $33,3 \%$ \\
\hline \multirow{4}{*}{$\begin{array}{l}\text { Área de co- } \\
\text { nhecimento }\end{array}$} & Matemática & 17 & $40,5 \%$ \\
\hline & $\begin{array}{l}\text { Ciências naturais } \\
\text { e da saúde }\end{array}$ & 7 & $16,7 \%$ \\
\hline & Diversas & 5 & $11,9 \%$ \\
\hline & Outras & 13 & $30,9 \%$ \\
\hline \multirow{4}{*}{$\begin{array}{l}\text { Público } \\
\text { alvo/nível } \\
\text { de ensino }\end{array}$} & $\begin{array}{l}\text { Professores } \\
\text { Educação básica }\end{array}$ & 15 & $35,7 \%$ \\
\hline & $\begin{array}{l}\text { Professores } \\
\text { Ensino superior }\end{array}$ & 9 & $21,4 \%$ \\
\hline & $\begin{array}{l}\text { Alunos de } \\
\text { Licenciatura }\end{array}$ & 6 & $14,3 \%$ \\
\hline & Outros & 12 & $28,6 \%$ \\
\hline \multirow{3}{*}{$\begin{array}{l}\text { Tipo de } \\
\text { intervenção }\end{array}$} & Formação continuada & 15 & $35,7 \%$ \\
\hline & Formação inicial & 5 & $11,9 \%$ \\
\hline & Nenhuma & 22 & $52,4 \%$ \\
\hline
\end{tabular}

Tabela 1. Delineamento metodológicos dos artigos empíricos ( $n=42$ artigos).

Ao relacionarmos a categoria Público alvo/nível de ensino, com a categoria Tipo de intervenção analisado nos artigos, identificamos o predomínio de estudos relacionados a professores da educação básica em intervenções de formação continuada (Tabela 1). Observa-se a ocorrência de poucos estudos com licenciandos em formação inicial. Cabe ressaltar que a maior parte dos estudos não estava relacionada à formação de professores propriamente dita, mas sim à utilização de métodos qualitativos como questionário/entrevistas e estudos de caso, com o objetivo de compreender como as tecnologias são utilizadas no ensino.

Além do delineamento metodológico, os artigos empíricos foram analisados quanto ao nível de utilização do modelo CTPC. Considerou-se possuir cunho teórico o artigo que citou o modelo CTPC na seção de introdução e ou discussão, como referencial teórico para a integração de tecnologia no ensino, sem utilizar ou propor instrumento e ou metodologia para investigar as bases de conhecimento envolvidas no modelo CTPC. Portanto, os artigos que utilizaram ou apresentaram alguma metodologia de análise para avaliar as bases de conhecimento de professores à luz do modelo CTPC, foram considerados de uso metodológico. A maioria dos artigos (91,5\%) abordou o modelo CTPC de forma conceitual (Uso teórico), na introdução ou discussão dos resultados obtidos, sem aplicá-lo metodologicamente. De fato, somente quatro dos 42 artigos procederam o uso metodológico (A17, A42, A49 e A50), indicando que embora os autores reconheçam a importância do modelo CTPC como referencial para a pesquisa em tecnologia educacional, as bases de conhecimento que envolvem a integração da tecnologia no ensino têm sido pouco investigadas.

Em um dos estudos metodológicos, Lopes (A24) analisou o processo de integração da tecnologia no ensino superior, por professores durante sua atuação em um curso de licenciatura em Matemática na modalidade a distância. A autora utilizou quatro categorias analíticas, que representavam as bases de conhecimento do professor que estão relacionadas ao uso da tecnologia (Conhecimento tecnológico, Conhecimento Pedagógico Tecnológico, Conhecimento Tecnológico do Conteúdo, Conhecimento Pedagógico Tecnológico do Conteúdo). A análise foi desenvolvida a partir de entrevistas em que se buscou identificar, nas falas dos professores, evidências de cada um dos tipos de conhecimentos propostos.

De forma semelhante, Espíndola, Struchiner e Giannella (A17) analisaram a experiência de professores universitários com a integração de tecnologias no ensino, em relação à articulação de seus conhecimentos pedagógicos, tecnológicos e de conteúdo. As autoras utilizaram entrevistas seguidas de técnica de análise de conteúdo. Foram consideradas cinco categorias de análise (Conhecimento de conteúdo, Conhecimento pedagógico, Conhecimento tecnológico, Conhecimento pedagógico do conteúdo e Conhecimento pedagógico tecnológico do conteúdo). 


\section{Discussão}

O Conhecimento Tecnológico Pedagógico do Conteúdo do professor é reconhecido como um fator crucial para que professores sejam bem sucedidos na integração de tecnologias em sua prática pedagógica [26], [27], [29]. Desde a publicação do artigo seminal "Technological Pedagogical Content Knowledge: A Framework for Teacher Knowledge" por Mishra e Koelher em 2006 [25], pesquisadores em várias partes do mundo, como Estados Unidos [36]-[39], Holanda [40], Turquia [41], [42], Austrália [43], Tailândia [44], Taiwan [45], [46], Singapura [47], [48], China [49] passaram a estudar, de forma empírica, as bases de conhecimento envolvidas neste modelo teórico.

Com o intuito de responder as perguntas de investigação aqui propostas, bem como compreender como o modelo teórico CTPC permeia a pesquisa acerca da integração da tecnologia no ensino no contexto Lusófono, são discutidos, a seguir, os resultados obtidos no presente estudo à luz da literatura internacional.

Pergunta 1 - Em que extensão o modelo CTPC está incorporado à produção científica acerca da integração da tecnologia no ensino, no contexto Lusófono?

A busca e seleção de artigos publicados em língua portuguesa resultou em 60 artigos relacionados de alguma forma ao modelo teórico CTPC. Observou-se que a quantidade de artigos publicados tem crescido nos últimos anos. Esse achado é semelhante ao obtido na revisão de literatura de Chai, Koh, e Tsai [31], que descreve aumento gradativo no número de publicações em língua inglesa sobre o modelo CTPC a partir do ano de 2006, alcançando 26 publicações em 2010. Por outro lado, ao tomarmos o conjunto de artigos teóricos somados aos empíricos, identificamos o predomínio do uso teórico deste referencial no contexto Lusófono. De fato, somente em quatro dos artigos se realizou a análise das bases de conhecimento dos professores. Outras revisões de literatura apresentaram resultados bastante diferentes. Chai et al. [31] e Voogt et al. [30] encontraram, respectivamente, 55 ( $74,3 \%$ do total) e 44 $(80 \%$ do total) artigos diretamente relacionados a avaliação das bases de conhecimento envolvidas no modelo CTPC.

Embora a maior parte dos artigos analisados aqui cite o modelo CTPC como referencial de integração de tecnologia no ensino, sem utilizá-lo para investigar as bases de conhecimento dos professores, os resultados obtidos indicam que o modelo CTPC já se encontra presente na produção científica em língua portuguesa. De forma geral, podemos afirmar que a produção científica no campo de tecnologia educacional, desenvolvida nesse contexto tem abordado o modelo CTPC de forma conceitual, e se encontra em um estágio inicial da pesquisa em comparação ao contexto internacional mais amplo.
Pergunta 2 - Quais os instrumentos e ou métodos de avaliação acerca das bases de conhecimento envolvidas no modelo CTPC estão disponíveis em língua portuguesa?

Dos 60 artigos analisados, quatro artigos empíricos (A17, A42, A49 e A50) e um teórico (A41) apresentaram processos metodológicos para avaliar as bases de conhecimento do professor. Entre eles, Sampaio e Coutinho (A41) traduziram do inglês para o português uma rubrica de avaliação de integração tecnológica em planos de ensino, indicando a preocupação das autoras em relação à utilização de instrumentos de análise baseados no modelo CTPC. Lopes (A24) e Espíndola, Struchiner e Giannella, (A17) utilizaram entrevistas seguidas de técnica de análise de conteúdo para analisar as bases de conhecimento do professor que estão relacionadas ao uso da tecnologia. $\mathrm{O}$ estabelecimento de categorias analíticas, baseadas no modelo CTPC, possibilitou por meio da análise das falas dos professores, identificar e compreender, os diferentes papéis e usos atribuídos às tecnologias na prática docente [50].

Ao analisarmos os cinco trabalhos que fizeram uso metodológico do modelo CTPC, cabe mencionar que nenhum deles utilizou procedimentos de validação para as técnicas utilizadas, como por exemplo taxa de concordância entre avaliadores (Inter-rater agreement) ou análise de validade e confiabilidade. $\mathrm{O}$ emprego dessas técnicas estatísticas, é recomendado no contexto internacional [30], [31], [32] demonstrando preocupação em desenvolver instrumentos e métodos que possuam validade e confiabilidade, importantes critérios de julgamento acerca da qualidade dos processos metodológicos empregados nas pesquisas educacionais [51].

Baseado no paradigma científico racionalista crítico, uma teoria ou modelo teórico, como no caso, o modelo CTPC, não passa de uma conjectura, uma antecipação justificada, que deve ser testada empiricamente [52]. A partir dessa lógica, o surgimento do modelo teórico CTPC descortinou todo um novo campo de pesquisa dentro da área da tecnologia educacional, na medida em que possibilitou aos investigadores desenvolverem novos métodos e instrumentos de avaliação, visando refutar ou validar, por meio de dados empíricos, a existência das referidas bases de conhecimento do professor. Desde então, a corrida acadêmica na comunidade internacional ocorre no sentido de validar estatisticamente instrumentos capazes de mensurar as bases de conhecimento de professores de diferentes disciplinas. Em relação aos instrumentos de pesquisa, destacase a busca pela validação de questionários de auto relato [30], [32]. Em revisão de literatura conduzida por Voogt et al. [30], foram encontrados 11 artigos relacionados à construção de itens de avaliação (assertivas) que professores deveriam concordar ou não em relação às suas bases de conhecimento. Oito desses artigos utilizaram procedimentos de validação estatística por meio de testes de validade e confiabilidade. Entre esses, o trabalho de Chai, Koh, e 
Tsai [53] foi o primeiro a descrever um instrumento que obteve validação estatística para as sete bases de conhecimento envolvidas no modelo CTPC. Nessa perspectiva, parece importante ampliar a realização de estudos que empreguem o modelo CTPC nos processos metodológicos da pesquisa educacional no contexto Lusófono. Adicionalmente, a utilização de instrumentos validados internacionalmente, contribuirá para um maior diálogo entre os resultados obtidos por diferentes grupos de pesquisa em todo o mundo, possibilitando comparações entre os diferentes contextos.

Outro achado relevante, diz respeito à formação de professores. Nenhum dos artigos aqui analisados lidou diretamente com a formação de professores para a incorporação no modelo CTPC em sua prática profissional. Tampouco se desenvolveu estratégia de formação de professores visando o aprimoramento das bases de conhecimento envolvidas no modelo CTPC. De forma geral, esses estudos investigaram os usos que os professores já fazem da tecnologia. A possibilidade de elaborar e desenvolver estratégias de formação inicial e continuada baseadas no modelo CTPC parece ser o limiar da pesquisa internacional atual. Após a busca por desenvolver métodos e instrumentos de avaliação, pesquisadores começaram a utilizá-los para avaliar suas intervenções de ensino. Por exemplo, Kopcha, Ottenbreit-Leftwich, Jung, e Baser [54] utilizaram um questionário de auto relato proposto por Schmidt et al [36] e uma rubrica de avaliação de integração tecnológica em planos de ensino criada e validada por Harris, Grandgenett, $\&$ Hofer [55]. O estudo foi realizado com 27 professores participantes de um curso, que tinha por objetivo desenvolver atividades instrucionais para o uso de tecnologia em sala de aula. Além de selecionar e criar recursos tecnológicos, relacionados aos conteúdos específicos, os professores planejaram uma aula que visava a integração de tecnologia pedagogia e conteúdo [54]. Já Koh \& Chai [56] utilizaram o questionário de auto relato proposto por Chai et al [53] e empregaram técnica de agrupamento (Cluster analysis) para avaliar as percepções de professores em relação às suas bases de conhecimento, antes e após um curso voltado para a elaboração de planos de aula que incorporassem o uso de tecnologias da informação e comunicação. Esses dois estudos recentes indicam como a pesquisa no campo de tecnologia educacional, especificamente acerca do modelo CTPC, vem sendo construída de forma consistente e lógica, tomando o passo anterior (a validação de instrumentos), como fundamento sobre o qual a pesquisa posterior se desenvolve (emprego de instrumentos validados para avaliar intervenções).

\section{Conclusões}

Os resultados obtidos e discutidos no presente estudo, indicam que a pesquisa acerca da integração de tecnologia no ensino com alicerce no modelo CTPC, no contexto Lusófono, encontra-se em um estágio inicial, no qual a importância do modelo para o campo da educação em geral e das tecnologias educacionais em especial é reconhecida. Esse reconhecimento se expressa pela quantidade crescente de estudos que cita o modelo na Introdução, como referencial ou na Discussão como um modelo importante para compreensão de seus resultados. No atual momento, os estudos se baseiam fundamentalmente em metodologias qualitativas, com instrumentos desenvolvidos independentemente pelos autores com objetivos específicos e portanto de pouca generalização possível.

No que tange a utilização do modelo CTPC na formulação de procedimentos metodológicos de pesquisa, a fim de mensurar as bases de conhecimento envolvidas no modelo teórico, observa-se um reduzido número de estudos que de fato aplicaram o modelo ao que ele pretende, ou seja, aferir os diferentes tipos de conhecimento de professores acerca da integração da tecnologia no ensino. A partir desta constatação, implicações acerca das possibilidades de pesquisa futura, com base no modelo teórico CTPC, são apresentadas.

\section{Implicações e pesquisa futura}

Baseado no pressuposto da psicologia cognitiva, de que o conhecimento está inscrito na mente das pessoas [57], o modelo CTPC prevê sete diferentes bases de conhecimento que professores deveriam possuir, visando realizar a integração de tecnologias na sua prática profissional. Este conjunto de conhecimentos constitui um importante e atual objeto de estudo no campo da pesquisa em tecnologia educacional. Ao levar em consideração, as revisões de literatura realizadas no contexto internacional, em língua inglesa, percebe-se que a pesquisa feita ao longo da última década, elaborou e validou instrumentos de avaliação das bases de conhecimento dos professores, seja por meio de instrumentos de auto relato (self report) ou por rubricas de análise de planos de aula. Além desses procedimentos, a análise de diálogos presenciais ou online em fóruns de discussão, bem como a observação de uma aula propriamente dita, podem ser importantes meios de identificação e avaliação das bases de conhecimento envolvidas no modelo CTPC.

O campo de pesquisa em tecnologia educacional necessita de uma maior investigação da validade do modelo CTPC no contexto Lusófono e, sobretudo, da "situação" dos professores de diferentes áreas do saber em relação às sete diferentes bases previstas pelo modelo. A pesquisa empírica sobre o modelo CTPC se beneficiaria da utilização de instrumentos validados internacionalmente, como questionários e rubricas de avaliação, o que permitiria o cotejamento de resultados obtidos com aqueles de outros países. Esse tipo de paralelo contribuiria para uma melhor compreensão e eventual reorientação das diversas políticas 
públicas voltadas para a utilização de tecnologias educacionais no ensino.

Por fim, entendemos que o modelo CTPC é uma importante abordagem teórica acerca da integração de tecnologia no ensino, entretanto não é a única. A obtenção de resultados empíricos de pesquisa acerca das bases de conhecimento de professores, no que tange a formulação teórica proposta no modelo CTPC, poderia ser complementada ou mesmo contraposta com outras abordagens teóricas que também lidam com a integração da tecnologia no ensino, como as formuladas por Rogers [58] e Davis [59]. Nesse sentido, o entendimento de como se dá o processo de difusão de inovações tecnológicas [58], bem como a análise de fatores externos sobre as crenças, atitudes e intenções dos indivíduos em aceitarem a tecnologia [59], poderia contribuir para aprimorar programas de formação que visem promover o aumento das habilidades dos professores em integrar tecnologias na prática docente.

Advogamos no presente estudo, a necessidade de se buscar encontrar caminhos metodológicos validados à luz do referencial teórico CTPC. A pesquisa futura, no campo da tecnologia educacional, poderá se consolidar na medida em que pesquisadores, formadores de professores e políticas públicas se apropriarem não somente de referenciais teóricos, mas em conjunto com eles, de instrumentos de avaliação que sejam eficazes em aferir adequadamente os resultados da aplicação de determinadas teorias na prática educacional.

\section{Agradecimentos}

O presente estudo foi apoiado por recursos de fomento de pesquisa obtidos junto à Fundação Carlos Chagas Filho de Amparo à Pesquisa do Estado do Rio de Janeiro (FAPERJ - E-26/110.546/2014) e ao Conselho Nacional de Pesquisa (CNPq - 481714/2013-8). Luiz Gustavo Ribeiro Rolando recebe bolsa de doutorado da Coordenação de Aperfeiçoamento de Pessoal de Nível Superior (CAPES). Gostaríamos de agradecer ao Dr. Gutemberg Gomes Alves e à Sra. Neusa H. S. P. Martins pela revisão do manuscrito.

\section{Apêndice 1}

\begin{tabular}{|c|c|c|}
\hline Código & Tipo de estudo & Artigo \\
\hline A01 & Empírico & $\begin{array}{l}\text { C. A. A. P. Abar. Educação Matemática na Era Digital. Rev. Iberoam. Ecucación Ma- } \\
\text { temática, 27:13-28, } 2011 \text {. }\end{array}$ \\
\hline A02 & Teórico & $\begin{array}{l}\text { M. M. Alves; J. Ribeiro; F. Simões. Universal Design for Learning (UDL): contributos } \\
\text { para uma escola de todos. Indagatio Didact., 5(4):121-146, } 2013 .\end{array}$ \\
\hline A03 & Empírico & $\begin{array}{l}\text { G. T. Barcelos; S. C. F. Batista. Uso de Aplicativos em Tablets no Estudo de Sistemas } \\
\text { Lineares: percepção de licenciandos em Matemática. In Nuevas Ideas en Informática } \\
\text { Educativa TISE, 2013, pp. 168-175. }\end{array}$ \\
\hline A04 & Empírico & $\begin{array}{l}\text { G. T. Barcelos; S. C. F. Batista. Interpretação geométrica de sistemas lineares: percep- } \\
\text { ção de licenciandos em matemática sobre o uso de aplicativos em tablets. In Encontro de } \\
\text { Educação Matemática, 2013, pp. 1-8. }\end{array}$ \\
\hline A05 & Teórico & $\begin{array}{l}\text { A. M. Cardoso; J. D. F. Azevedo; R. X. Martins. Histórico e tendências de aplicação das } \\
\text { tecnologias no sistema educacional brasileiro. Colabor@, 8(30):1-11, } 2014 .\end{array}$ \\
\hline A06 & Empírico & $\begin{array}{l}\text { F. A. Costa; C. Rodriguez. O desenho de estratégias de trabalho com TIC com base no } \\
\text { conhecimento de professores experientes: o caso das artes. In II Congresso Internac- } \\
\text { ional TIC e Educação, 2012, pp. 2739-2752. }\end{array}$ \\
\hline A07 & Empírico & $\begin{array}{l}\text { J. P. da Costa; A. C. C. Costa; S. Peres; G. A. P. B. Teixeira. Desvendando a ecologia } \\
\text { local: Atividades interativas. Rev. Bras. Ensino Ciência e Tecnol., 4(3):119-134, } 2011 .\end{array}$ \\
\hline A08 & Empírico & $\begin{array}{l}\text { N. M. L. da Costa; M. E. B. B. Prado. Formação continuada e uma abordagem explora- } \\
\text { tório investigativa em geometria espacial de posição. In VII Congreso Iberomericano de } \\
\text { Educación Matemática, 2013, pp. 5143-5150. }\end{array}$ \\
\hline
\end{tabular}




\begin{tabular}{|c|c|c|}
\hline A09 & Teórico & $\begin{array}{l}\text { C. P. Coutinho. TPACK: em busca de um referencial teórico para a formação de profes- } \\
\text { sores em Tecnologia Educativa. Paidei@, 2(4):1-18, } 2011 .\end{array}$ \\
\hline A10 & Teórico & $\begin{array}{l}\text { C. P. Coutinho; A. Sousa. Conteúdos digitais (interactivos) para educação: questões de } \\
\text { nomenclatura, reutilização, qualidade e usabilidade. Paidei@, (2)2:1-14, } 2009 .\end{array}$ \\
\hline A11 & Empírico & $\begin{array}{l}\text { E. J. Dias; C. F. Araujo Jr. Mobile learning no ensino de matemática: um framework } \\
\text { conceitual para uso dos tablets na educação básica. In Encontro de Produção Discente } \\
\text { PUCSP, 2012, pp. 1-9. }\end{array}$ \\
\hline A12 & Teórico & $\begin{array}{l}\text { F. A. da S. Dias; N. M. L. da Costa. Formação continuada de professores da educação } \\
\text { básica do estado de São Paulo e a integração de tecnologias digitais ao currículo de ma- } \\
\text { temática, In VII Congreso Iberomericano de Educación Matemática, 2013, pp. 5119- } \\
5126 .\end{array}$ \\
\hline A13 & Empírico & $\begin{array}{l}\text { J. A. de O. Duarte. Tecnologias para desenvolver o pensamento algébrico. In II Con- } \\
\text { gresso Internacional TIC e Educação, 2012, pp. 1927-1943. }\end{array}$ \\
\hline A14 & Empírico & $\begin{array}{l}\text { J. Duarte; J. Brocardo; J. P. da Ponte. Tecnologias e pensamento algébrico: conhecimen- } \\
\text { tos e práticas de duas professoras de matemática. In Encontro de Investigação em Edu- } \\
\text { cação Matemática, 2011, pp. 71-86. }\end{array}$ \\
\hline A15 & Empírico & $\begin{array}{l}\text { M. B. de Espíndola; T. R. Giannella; M. Struchiner. Análise de ambientes virtuais de } \\
\text { aprendizagem construídos por professores universitários da área de ciências e da saúde. } \\
\text { In VI Encontro nacional de pesquisa em educação e Ciências, 2007, pp. 1-10. }\end{array}$ \\
\hline A16 & Empírico & $\begin{array}{l}\text { M. B. De Espíndola; M. Struchiner; T. R. Giannella. Tecnologias de Informação e Co- } \\
\text { municação no Ensino Superior: Análise das Percepções de Professores das Âreas das Ci- } \\
\text { ências e da Saúde sobre o Processo de Integração da Ferramenta Constructore em suas } \\
\text { Práticas Educativas. In Encontro Nacional de Pesquisa em Educação em Ciências, } \\
\text { 2011, pp. 1-13. }\end{array}$ \\
\hline A17 & Empírico & $\begin{array}{l}\text { M. B. de Espíndola; M. Struchiner; T. R. Giannella. Análise das experiências de integra- } \\
\text { ção de tecnologias de informação e comunicação no ensino superior por professores das } \\
\text { áreas de ciências e da saúde: contribuições do conhecimento pedagógico -tecnológico do } \\
\text { conteúdo. In Encontro nacional de pesquisa em educação em ciências, 2009, pp. 1-12. }\end{array}$ \\
\hline A18 & Empírico & $\begin{array}{l}\text { A. da C. Esquincalha; C. A. A. P. Abar. Impressões iniciais sobre as práticas de tutoria } \\
\text { em um curso a distância para professores de matemática. In Encontro de Produção Dis- } \\
\text { cente PUCSP, 2012, pp. 1-11. }\end{array}$ \\
\hline A19 & Empírico & $\begin{array}{l}\text { Á. Faria; P. M. Faria; M. A. Ramos. Formação e desenvolvimento profissional docente } \\
\text { em rede: entre o presencial e o online. Educ. em Perspect., 4(2):374-395, } 2013 .\end{array}$ \\
\hline A20 & Empírico & $\begin{array}{l}\text { A. Fernandes; M. Meirinhos. A integração curricular das TIC: diagnóstico de uma escola } \\
\text { do Ensino Básico e Secundário. In Conferência Ibérica Inovação na Educação com TIC, } \\
\text { 2012, pp. 395-411. }\end{array}$ \\
\hline A21 & Empírico & $\begin{array}{l}\text { C. A. Figueiredo; L. C. Contreras. A função quadrática: variação , transparência e duas } \\
\text { tipologias de exemplos. Av. Investig. en Educ. Matemática, 3:45-68, } 2013 \text {. }\end{array}$ \\
\hline A22 & Empírico & $\begin{array}{l}\text { E. S. Goudouris; T. R. Giannella; M. Struchiner. Tecnologias de informação e comuni- } \\
\text { cação e ensino semipresencial na educação médica. Rev. Bras. Educ. Med., 37(3)396- } \\
\text { 407, } 2013 \text {. }\end{array}$ \\
\hline A23 & Empírico & $\begin{array}{l}\text { J. Lima; W. Rochadel; J. Silva. Utilização da Experimentação Remota Móvel em Disci- } \\
\text { plina de Física do Ensino Médio. In International Conference on Interactive Computer } \\
\text { aided Blended Learning, 2013, pp. 255-260. }\end{array}$ \\
\hline A24 & Empírico & $\begin{array}{l}\text { J. P. Lopes. A tecnologia na ótica dos professores: análise da integração entre conheci- } \\
\text { mentos pedagógicos, tecnológicos e do conteúdo. In XIII Conferência Interamericana de } \\
\text { Educaçao Matemática, 2011, pp. 1-11. }\end{array}$ \\
\hline
\end{tabular}




\begin{tabular}{|c|c|c|}
\hline A25 & Empírico & $\begin{array}{l}\text { A. A. Oliveira. Ensinar e aprender história com lições e testes Moodle. História \& En- } \\
\text { sino, 18(1):07-25, } 2012 \text {. }\end{array}$ \\
\hline A26 & Teórico & $\begin{array}{l}\text { G. D. L. R. Palis. O conhecimento tecnológico, pedagógico e do conteúdo do professor } \\
\text { de Matemática. Educ. Matemática Pesqui., 12(3):432-451, } 2010 .\end{array}$ \\
\hline A27 & Empírico & $\begin{array}{l}\text { S. C. B. Piconez; R. H. R. Nakashima. Design integrado e articulação dialética das di- } \\
\text { mensões avaliativas na EAD. In Simpósio Internacional de Educação a Distância, 2012, } \\
\text { pp. 1-13. }\end{array}$ \\
\hline A28 & Empírico & $\begin{array}{l}\text { S. C. do A. Porto. Jogos Envolvendo Números Inteiros: Um Episódio na Classe de San- } \\
\text { dra. In XVII Encontro Nacional de Estudantes de Pós-Graduação em Educação Mate- } \\
\text { mática, 2013, pp. 1-12. }\end{array}$ \\
\hline A29 & Teórico & $\begin{array}{l}\text { A. B. Powell; M. A. Bairral. Investigando a aprendizagem matemática de alunos e pro- } \\
\text { fessores colaborando e resolvendo problemas em um cenário virtual. In VI Congreso } \\
\text { Iberomericano de Educación Matemática, 2009, pp. 1-6. }\end{array}$ \\
\hline A30 & Empírico & $\begin{array}{l}\text { M. E. B. B. Prado; N. M. L. da Costa. O processo de apropriação das TIC e a reconstru- } \\
\text { ção de novas práticas no ensino de matemática. In VII Congreso Iberomericano de Edu- } \\
\text { cación Matemática, 2013, pp. 6973-6980. }\end{array}$ \\
\hline A31 & Teórico & $\begin{array}{l}\text { J. L. Ramos. Repensar as TIC na Educação. O Professor como Agente Transformador. } \\
\text { Educ. Formação Tecnol., 6(1):94-98, } 2013 .\end{array}$ \\
\hline A32 & Empírico & $\begin{array}{l}\text { P. Ramos; T. Rabetti; G. Miriam. A Pesquisa Baseada em Design em Artigos Científicos } \\
\text { Sobre o Uso de Ambientes de Aprendizagem Mediados Pelas Tecnologias da Informa- } \\
\text { ção e da Comunicação no Ensino de Ciências. Alexandria, 3(1):77-102, } 2010 .\end{array}$ \\
\hline A33 & Empírico & $\begin{array}{l}\text { A. M. M. da Rocha; C. P. Coutinho. A utilização das TIC por professores de educação } \\
\text { visual: um estudo em escolas com 3o ciclo de Paços de Ferreira, Paredes e Penafiel. In } \\
\text { VII Conferência Internacional de TIC na Educação, 2011, pp. 189-200. }\end{array}$ \\
\hline A34 & Empírico & $\begin{array}{l}\text { K. D. M. Rocha; M. Bittar. Um estudo do processo de gênese instrumental do software } \\
\text { superlogo por acadêmicos de um curso de pedagogia para o ensino de matemática. EM } \\
\text { TEIA - Rev. Educ. Matemática e Tecnológica Iberoam., 3(3):1-16, } 2012 \text {. }\end{array}$ \\
\hline A35 & Empírico & $\begin{array}{l}\text { K. de M. Rocha; M. Bittar. Uma discussão sobre os conhecimentos e os processos vi- } \\
\text { venciados por professores tendo em vista a integração da tecnologia em sua prática. In } \\
\text { VII Congreso Iberomericano de Educación Matemática, 2013, pp. 4740-4747. }\end{array}$ \\
\hline A36 & Teórico & $\begin{array}{l}\text { L. Salema; T. Cardoso. Curriculum e Novos Media na Educação em Línguas. In II Con- } \\
\text { gresso Internacional TIC e Educação, 2012, pp. 1425-1442. }\end{array}$ \\
\hline A37 & Teórico & $\begin{array}{l}\text { L. Salema. Tecnologia, educação a distância e aprendizagem de línguas. Rev. Linhas, } \\
\text { 13(2):123-139, } 2012 \text {. }\end{array}$ \\
\hline A38 & Empírico & $\begin{array}{l}\text { D. F. Salvador; L. G. R. Rolando; R. F. R. Rolando. Aplicação do modelo de conheci- } \\
\text { mento tecnológico, pedagógico do conteúdo (TPCK) em um programa on-line de forma- } \\
\text { ção continuada de professores de Ciências e Biologia. Rev. Electrónica Investig. en } \\
\text { Educ. en Ciencias, } 5(2): 31-43,2010 \text {. }\end{array}$ \\
\hline A39 & Empírico & $\begin{array}{l}\text { D. F. Salvador; R. F. R. Rolando; L. G. R. Rolando. Colaborar para aprender e avaliar } \\
\text { para formar: Um relato de experiência na formação continuada de professores de biolo- } \\
\text { gia. Rev. Bras. Aprendiz. Aberta e a Distância, 11:35-48, } 2012 \text {. }\end{array}$ \\
\hline A40 & Empírico & $\begin{array}{l}\text { P. Sampaio; C. Coutinho. Uma perspectiva sobre a formação contínua em TIC: essencial } \\
\text { ou apenas uma acreditação? In IX Colóquio sobre Questões Curriculares/V Colóquio } \\
\text { Luso-Brasileiro, 2010, pp. 3975-3984. }\end{array}$ \\
\hline A41 & Teórico & $\begin{array}{l}\text { P. Sampaio; C. Coutinho. Avaliação do TPACK nas atividades de ensino e aprendiza- } \\
\text { gem: um contributo para o estado da arte. Rev. Educ., 6(3):39-55, } 2012 .\end{array}$ \\
\hline
\end{tabular}




\begin{tabular}{|c|c|c|}
\hline A42 & Teórico & $\begin{array}{l}\text { P. Sampaio; C. Coutinho. Ensinar com Tecnologia, Pedagogia e Conteúdo. Paidei@, } \\
\text { 5(8):01-17, } 2013 .\end{array}$ \\
\hline A43 & Empírico & $\begin{array}{l}\text { P. Sampaio; C. Coutinho. Formação continuada de professores: integraçao das tic. } \\
\text { FAED - Rev. da Fac. Educ., 9(15):139-151, } 2011 .\end{array}$ \\
\hline A44 & Empírico & $\begin{array}{l}\text { A. A. De Souza; D. O. Xavier; B. F. Tormin; F. A. Silva. Sistema de seleção remota: } \\
\text { uma ferramenta para processos de seleção online. In Simpósio Internacional de Educa- } \\
\text { çao a Distância, 2006, pp. 1-14. }\end{array}$ \\
\hline A45 & Empírico & $\begin{array}{l}\text { M. E. Weinert; S. A. de Lima; I. R. Gravonski; H. Moreira. O uso das Tecnologias de } \\
\text { Informação e Comunicação no cotidiano escolar das séries iniciais: panorama inicial. } \\
\text { Rev. Bras. Ensino Ciência e Tecnol., 4(3):50-72, } 2011 .\end{array}$ \\
\hline A46 & Teórico & $\begin{array}{l}\text { W. M. Yonezawa; A. Konsianski; P. L. Filho. Introdução ao desenvolvimento de jogos } \\
\text { digitais para professores de matemática utilizando programação visual. In XI Encontro } \\
\text { Nacional de Educação Matemática, 2013, pp. 1-7. }\end{array}$ \\
\hline A47 & Teórico & $\begin{array}{l}\text { P. Sampaio; C. Coutinho. Integração do TPACK no processo de ensino/ aprendizagem } \\
\text { da matemática. Paidei@, 6(10):1-20, } 2014 .\end{array}$ \\
\hline A48 & Empírico & $\begin{array}{l}\text { B. D. da Silva; A. M. Araújo; C. M. Vendramini; R. X. Martins; N. M. Piovezan; E. Pra- } \\
\text { tes; A. S. Dias; L. S. Almeida; M. C. R. A. Joly. Aplicação e uso de tecnologias digitais } \\
\text { pelos professores do ensino superior no Brasil e em Portugal. Educ. Formação Tecnol., } \\
\text { 7(1):3-18, } 2014 \text {. }\end{array}$ \\
\hline A49 & Empírico & $\begin{array}{l}\text { K. D. M. Rocha; M. Bittar. Uma articulação teórica para análise de um processo de inte- } \\
\text { gração da tecnologia na prática pedagógica. Nuances Estud. sobre Educ., 25(2):109- } \\
\text { 126, } 2014 .\end{array}$ \\
\hline A50 & Empírico & $\begin{array}{l}\text { C. A. A. P. Abar; R. E. M. Nifoci. Conhecimentos revelados nas Narrativas de professo- } \\
\text { res de Matemática sobre o uso de Objetos de Aprendizagem. Rev. Iberoam. Ecucación } \\
\text { Matemática, 38:51-68, } 2014 .\end{array}$ \\
\hline A51 & Teórico & $\begin{array}{l}\text { H. Rocha. Utilização, uso ou integração da tecnologia: Contributo para a clarificação de } \\
\text { um conceito. In XXV Seminário de Investigação em Educação Matemática, 2014, pp. } \\
\text { 141-153. }\end{array}$ \\
\hline A52 & Empírico & $\begin{array}{l}\text { R. X. Martins; L. B. Serafim. Percepção de estudantes quanto aos diferenciadores do de- } \\
\text { sempenho escolar em cursos de licenciatura a distância. In } 20^{\circ} \text { Congresso Internacional } \\
\text { ABED de Educação a Distância, 2014, pp. 1-10. }\end{array}$ \\
\hline A53 & Empírico & $\begin{array}{l}\text { K. R. de P. Nazario; M. R. G. de Oliveira. Curso formação docente em EaD: analise par- } \\
\text { cial dos dados de um questionário online. In Simpósio Internacional de Educação a Dis- } \\
\text { tância, 2014, pp. 1-13. }\end{array}$ \\
\hline A54 & Empírico & $\begin{array}{l}\text { F. H. A. Rossit; M. R. G. de Oliveira. Base de conhecimento docente para o ensino de } \\
\text { teclado a distância. In Simpósio Internacional de Educação a Distância, 2014, pp. 1-12. }\end{array}$ \\
\hline A55 & Empírico & $\begin{array}{l}\text { R. X. Martins; U. A. Leitão; A. J. de C. Silva. Pesquisas em educação a distância: pano- } \\
\text { rama da produção apresentada no ESUD entre } 2010 \text { e 2013. In XI Congresso Brasileiro } \\
\text { de Ensino Superior a Distância, 2014, pp. 2759-2773. }\end{array}$ \\
\hline A56 & Teórico & $\begin{array}{l}\text { M. B. de Espíndola; N. G. Gomes; G. G. S. Teixeira; J. Schiller; C. C. D. Búrigo; P. B. } \\
\text { Pereira; M. Espindula. Formação docente para o ensino superior mediado por tecnolo- } \\
\text { gias de informação e comunicação: perspectivas teórico metodológicas do laboratório de } \\
\text { novas tecnologias - lantec/ced/ufsc. In XI Congresso Brasileiro de Ensino Superior a } \\
\text { Distância, 2014, pp. 3289-3301. }\end{array}$ \\
\hline
\end{tabular}




\begin{tabular}{|l|l|l|}
\hline A57 & Teórico & $\begin{array}{l}\text { W. R. Padilha. O professor de Matemática da Educação Básica e o processo de apropria- } \\
\text { ção das tecnologias móveis. In Encontro Brasileiro de Estudantes de Pós-Graduação em } \\
\text { Educação Matemática, 2014, pp. 1-10. }\end{array}$ \\
\hline A58 & Empírico & $\begin{array}{l}\text { F. M. Santos; G. T. Barcelos; S. C. F. Batista. Formação de Professores de Matemática: } \\
\text { Avaliação de Recursos Digitais. In XIX Workshop de Informática na Escola, 2014, pp. } \\
1-10 .\end{array}$ \\
\hline A59 & Empírico & $\begin{array}{l}\text { E. Educacional, “Interação, interatividade e comunicação no processo de mediação pe- } \\
\text { dagógica na educação on-line," Educere Educ., vol. 9, no. 18, pp. 765-779, 2014. }\end{array}$ \\
\hline A60 & Teórico & $\begin{array}{l}\text { G. A. Zimmermann; L. A. de Azevedo; V. G. Pedroso. Os institutos federais de educa- } \\
\text { ção, ciência e tecnologia, uma nova institucionalidade, e sua educação profissional, cien- } \\
\text { tífica e tecnológica: uma nova-velha prática educacional? In IV Seminário Nacional de } \\
\text { Educação Profissional e Tecnológica, 2008, pp. 1-23. }\end{array}$ \\
\hline
\end{tabular}

\section{Referências}

[1] M. Castells. The rise of the network society. Information age: economy, society and culture, Volume 1. Oxford: Blackwell, 1996.

[2] M. Castells; G. Cardoso. The network society: from knowledge to policy. Washington, DC: Johns Hopkins Center for Transatlantic Relations, 2005.

[3] P. Lévy. Cyberculture. Minneapolis, MN: University of Minnesota Press, 2001.

[4] K. J. Kaufman. 21 Ways to 21 st Century Skills: Why Students Need Them and Ideas for Practical Implementation. Kappa Delta Pi Record. 49:7883, 2013.

[5] J. Voogt; O. Erstad; C. Dede; P. Mishra. Challenges to learning and schooling in the digital networked world of the 21 st century. Journal of Computer Assisted Learning, 29(5):403-413, 2013.

[6] S. Martin; G. Diaz; E. Sancristobal; R. Gil; M. Castro; J. Peire. New technology trends in education: Seven years of forecasts and convergence. Computers \& Education, 57(3):1893-1906, 2011. doi: 10.1016/j.compedu.2011.04.003

[7] P. Goodyear; S. Banks; V. Hodgson; D. McConnell. Advances in research on networked learning, vol. 7. Dordrecht, Netherlands: Kluwer Academic Publishers, 2004.

[8] F. C. A. Carvalho; G. B. Ivanoff. Tecnologias que educam: ensinar e aprender com tecnologias da informação e comunicação. São Paulo: Pearson
Prentice Hall, 2010.

[9] BECTA. What the research says about using ICT in science. Report to the BECTA ICT Research network, 2003.

[10] C. Crook. Web 2.0 technologies for learning: The current landscape-opportunities, challenges and tensions. British Educational Communications and Technology Agency (BECTA) Report: Web 2.0 technologies for learning at Key Stages 3 and 4, pp. 1-25, 2008.

[11] UNESCO. Unesco ICT competency framework for teachers. Paris, France, 2011.

[12] UNESCO. ICT competency standards for teachers. Paris, France, 2008.

[13] J. D. Bransford; A. L. Brow; R. R. Cocking. Como as pessoas aprendem: cérebro, mente, experiencia e escola. 2007.

[14] S. W.-Y. Lee; C.-C. Tsai; Y.-T. Wu; M.-J. Tsai; T.-C. Liu; F.-K. Hwang; C.-H. Lai; J.-C. Liang; H.-C. Wu; C.-Y. Chang. Internet-based Science Learning: A review of journal publications. International Journal of Science Education, 33(14):1893-1925, 2011.

[15] L. G. R. Rolando; D. F. Salvador; A. H. S. Souza; M. R. M. P. Luz. Learning with their peers: Using a virtual learning community to improve an inservice Biology teacher education program in Brazil. Teaching and Teacher Education, 44:4455, 2014. doi:10.1016/j.tate.2014.07.010

[16] D. F. Salvador; J. Magarão; M. Struchiner; T. Giannella. Laboratório Móvel de Informática (LMI) para o ensino de Ciências e Matemática: uma abordagem de utilização de um computador por aluno na sala de aula. In: Encontro nacional de 
pesquisa em educação em ciências, 2011, pp. 0113.

[17] A. Balanskat; R. Blamire; S. Kefala. The ICT impact report: A review of studies of ICT impact on schools in Europe, 2006.

[18] J. Osborne; S. Hennessy, Literature Review in Science Education and the Role of ICT: Promise, Problems and Future Directions, Bristol: United Kingdom, 2003.

[19] CNE. Resolução CNE/CP no 1, de 18 de fevereiro de 2002. Institui Diretrizes Curriculares Nacionais para a Formação de Professores da Educação Básica, em nível superior, curso de licenciatura, de graduação plena., Brasília, DF, 2002.

[20] Brasil. LEI No 13.005, DE 25 JUNHO DE 2014. Plano Nacional de Educação. Brasília, DF, pp. 115, 2014.

[21] CONAE. Construindo o Sistema Nacional Articulado de Educação: o Plano Nacional de Educação, diretrizes e estratégias; documento Final., Brasília, DF, 2010.

[22] L. G. R. Rolando; D. F. Salvador; M. R. M. P. Luz. The use of internet tools for teaching and learning by in-service biology teachers: A survey in Brazil. Teaching and Teacher Education, 34:46-55, 2013. doi:10.1016/j.tate.2013.03.007

[23] L. G. R. Rolando; R. F. R. R. Vasconcellos; E. L. Moreno; D. F. Salvador; M. R. M. P. Da Luz. Integration between Internet and Chemistry Teaching Practice. Revista Virtual de Química, 7(3):864-879, 2015. doi:10.5935/19846835.20150044

[24] H. H. O. de M. Couto; L. A. C. de R. Mídias na Educação: discurso oficial nos discursos de professores egressos de um Programa de Formação Continuada. Revista Brasileira de Informática na Educação, 21(3):85-99, 2013. doi:10.5753/RBIE.2013.21.03.85

[25] P. Mishra; M. J. Koehler. Technological Pedagogical Content Knowledge: A Framework for Teacher Knowledge. Teachers College Record, 108(6):1017-1054, 2006.

[26] M. J. Koehler; P. Mishra. Introducing TPCK. In: The handbook of technological pedagogical content knowledge (TPCK) for educators, A. C. on I. and Technology, Ed. American Association of Colleges of Teacher Education and Rougledge, NY, New York, 2008, pp. 3-29.

[27] M. Koehler; P. Mishra; W. Cain. What Is Technological Pedagogical Content Knowledge
(TPACK)? Journal of Education, 193(3):13-19, 2013.

[28] L. S. Shulman. Knowledge and Teaching: Foundations of New Reform. Harvard Educational Review, 57(1):01-21, 1987.

[29] Y.-T. Wu. Research trends in technological pedagogical content knowledge (TPACK) research: A review of empirical studies published in selected journals from 2002 to 2011. British Journal of Educational Technology, 44(3):73-76, 2013. doi: 10.1111/j.1467-8535.2012.01349.x

[30] J. Voogt; P. Fisser; N. Pareja Roblin; J. Tondeur; J. van Braak. Technological pedagogical content knowledge - a review of the literature. Journal of Computer Assisted Learning, 29(2):109-121, 2013. doi: 10.1111/j.1365-2729.2012.00487.x

[31] C. Chai; J. Koh; C. Tsai. A review of Technological Pedagogical Content Knowledge. Education Technology and Society, 16(2):31-51, 2013.

[32] M. Koehler; T. Shin; P. Mishra. How do we measure TPACK? Let me count the ways. In: Educational Technology, Teacher Knowledge, and Classroom Impact: A Research Handbook on Frameworks and Approaches, R. N. Ronau, C. R. Rakes, and M. L. Niess, Eds. United States of America: Information Science Reference, 2012, pp. 16-31.

[33] R. F. Sampaio; M. C. Mancini. Estudos de revisão sistemática: um guia para síntese criteriosa da evidência científica. Revista Brasileira de Fisioterapia, 11(1):83-89, 2007.

[34] H. Cooper; L. Hedges. Research synthesis as a scientific process. In: The handbook of research synthesis and meta-analysis, 2nd ed., H. Cooper, L. V. Hedges, and J. C. Valentine, Eds. Russell Sage Foundation, 2009, pp. 3-17.

[35] B. Kitchenham. Procedures for performing systematic reviews. Technical report, Keele University and NICTA, 2004.

[36] D. A. Schmidt; E. Baran; A. D. Thompson; P. Mishra; M. J. Koehler; T. S. Shin. Technological Pedagogical Content Knowledge (TPACK): The Development and Validation of an Assessment Instrument for Preservice Teachers. Journal of Research on Technology in Education, 42(2):123-149, 2009.

[37] C. R. Graham; N. Burgoyne; P. Cantrell; L. Smith; L. St. Clair; R. Harris. TPACK Development in Science Teaching: Measuring the 
TPACK Confidence of Inservice Science Teachers. TechTrends, 53(5):70-79, 2009.

[38] C. R. Graham. Theoretical considerations for understanding technological pedagogical content knowledge (TPACK). Computers \& Education, 57(3):1953-1960, 2011. doi: 10.1016/j.compedu.2011.04.010

[39] M. Hofer; N. Grandgenett; J. Harris; K. Swan. Testing a TPACK-based technology integration observation instrument. In: Teacher Education Faculty Proceedings \& Presentations, 2011, pp. 19.

[40] B. Rienties; N. Brouwer; S. Lygo-Baker. The effects of online professional development on higher education teachers' beliefs and intentions towards learning facilitation and technology. Teaching and Teacher Education, 29:122-131, 2013. doi: 10.1016/j.tate.2012.09.002

[41] S. Pamuk; M. Ergun; R. Cakir; H. B. Yilmaz; C. Ayas. Exploring relationships among TPACK components and development of the TPACK instrument. Education and Information Technologies, Sep. 2013. doi: 10.1007/s10639-013-9278-4

[42] S. Pamuk. Understanding preservice teachers' technology use through TPACK framework. Journal of Computer Assisted Learning, 28(5):425-439, 2012. doi: 10.1111/j.13652729.2011.00447.x

[43] G. Finger; R. Jamieson-Proctor; P. Albion. Beyond Pedagogical Content Knowledge: The Importance of TPACK for Informing Preservice Teacher Education in Australia Introduction Moving Beyond PCK to TPACK to Design. In: WCC 2010: 21st World Computer Congress: Key Compentencies in the Knowledge Society, 2010, pp. 20-23.

[44] N. Srisawasdi. The Role of TPACK in Physics Classroom: Case Studies of Preservice Physics Teachers. Procedia - Social and Behavioral Sciences, 46:3235-3243, 2012. doi: 10.1016/j.sbspro.2012.06.043

[45] S. Jang; M. Tsai. Exploring the TPACK of Taiwanese secondary school science teachers using a new contextualized TPACK model. Australasian Journal of Educational Technology, 29(4):566580, 2013.

[46] Y.-H. Chen; S.-J. Jang. Interrelationship between Stages of Concern and Technological, Pedagogical, and Content Knowledge: A study on Taiwanese senior high school in-service teachers. Computers in Human Behavior, 32:79-91, 2014. doi:

\subsection{6/j.chb.2013.11.011}

[47] J. H. L. Koh; C. S. Chai; C.-C. Tsai. Examining practicing teachers' perceptions of technological pedagogical content knowledge (TPACK) pathways: a structural equation modeling approach. Instructional Science, Jun. 2012. doi: 10.1007/s11251-012-9249-y

[48] J. H. Ling Koh; C. S. Chai; L. Y. Tay. TPACKin-Action: Unpacking the Contextual Influences of Teachers' Construction of Technological Pedagogical Content Knowledge (TPACK). Computers \& Education, 78:20-29, 2014. doi: 10.1016/j.compedu.2014.04.022

[49] G. Sang; J. Tondeur; C. S. Chai; Y. Dong. Validation and profile of Chinese pre-service teachers' technological pedagogical content knowledge scale. Asia-Pacific Journal of Teacher Education, 37-41, $2014 . \quad$ doi: 10.1080/1359866X.2014.960800

[50] M. B. de Espíndola; M. Struchiner; T. R. Giannella. Análise das experiências de integração de tecnologias de informação e comunicação no ensino superior por professores das áreas de ciências e da saúde: contribuições do conhecimento pedagógico -tecnológico do conteúdo. In: Encontro nacional de pesquisa em educação em ciências, 2009, pp. 1-12.

[51] J. Fraenkel; N. Wallen. How to design and evaluate research in education, 7th ed. New York: McGraw-Hill, 2008.

[52] K. R. Popper. Ciência: Conjecturas e Refutações, in Conjecturas e Refutações, Brasília: Editora da UnB, 1980, pp. 1-27.

[53] C. S. Chai; J. H. ling Koh; C.-C. Tsai. Exploring the Factor Structure of the Constructs of Technological, Pedagogical, Content Knowledge (TPACK). The Asia-Pacific Education Researcher, 20(3):595-603, 2011.

[54] T. J. Kopcha; A. Ottenbreit-Leftwich; J. Jung; D. Baser. Examining the TPACK framework through the convergent and discriminant validity of two measures. Computers \& Education, 78:8796, 2014. doi: 10.1016/j.compedu.2014.05.003

[55] J. Harris; N. Grandgenett; M. Hofer. Testing a TPACK-Based Technology Integration Assessment Rubric. In: Proceedings of Society for Information Technology \& Teacher Education International Conference, 2010, pp. 3833-3840.

[56] J. H. L. Koh; C. S. Chai. Teacher clusters and their perceptions of technological pedagogical 
content knowledge (TPACK) development through ICT lesson design. Computers \& Education, 70:222-232, 2014. doi: 10.1016/j.compedu.2013.08.017

[57] R. J. Sternberg. Cognitive Psychology, Fourth Edi. United States of America: Thomson Wardsworth, 2006.

[58] E. M. Rogers. Diffusion of Innovations, 4th Editio. New York: Free Press, 1995.

[59] F. Davis. Perceived usefulness, perceived ease of use, and user acceptance of information technology. MIS Quarterly, 13(3):319-340, 1989. 